\title{
Cancer recurrence prevention program was scientifically carried out by utilizing dynamic tumor marker combination
} assay

\author{
Tsuneo Kobayashi* \\ International cancer prevention center, Chiba city, Mihama-ku Takasu 3-21-1, Japan
}

\begin{abstract}
Background: Standard therapy are saying that cancer recurrence prevention is difficult. Shelton L.N. reported that cancer cell is grown up in the presence of both glucose and glutamine. So, dietary energy restriction (DER) is useful tool of recurrence prevention. As the second item, I have exploited herbal medicine (Sun Advance) which inhibit the hallmarks of carcinogenesis. Now I have employed detoxifying therapy. As the 4th method, high dose of vitamin C therapy which promotes the increasing of hydro-peroxide in cancer cell. I applied these 4 combinations of intervention method on the high risk of postoperative cancer patients.
\end{abstract}

Method: Among 500 post-operative patients, I have selected 131 candidates who were classified into tumor stage IV and tumor stage V. Among them, 104 members are intervened, and 27 patients are classified into control group.

Results: In control group, 55\% have tendency to aggravating change. Among intervened 104 members, $7.7 \%$ are not prevented.

\section{Introduction}

They said that there is no correct barometer for cancer prevention and recurrence prevention. So as to solve this problem, we have reported tumor marker combination assay (TMCA) in Cancer (1994) [1]. By utilizing TMCA, I have classified cancer risk assessment into 5 tumor stages among 500 postoperative cancer patients and I have selected 131 high risk group, who are classified into TSIV and TSV. I have intervened by 4 methods against high risk groups. Shelton LN et al. [2-4] reported that VM-M3 cancer cell is grown up in the presence of both glucose and glutamine. So, wise dietary energy restriction (DER) has induced from this knowledge as the nutritional counter measure for cancer prevention [5-8]. Youngman LD reported that the combination of carcinogen and high protein diet highly promote the carcinogenesis. As the second item, I have exploited herbal medicine (Sun Advance) [9-10] which inhibit selectively mitochondrial oxygen respiration on cancer cell and which inhibit its hallmarks of carcinogenesis of culture cell. Now I have employed detoxifying therapy which is utilized in old type of Japanese therapy as CAM, which is mainly composed of Nishi Type (Orthopedic Traction Apparatus: WA: 57B (686) Yamato (KK, Ehime prefecture, Japan) of exercise and sweeping of intestinal canal which eliminate immunosuppressive substances from bloods and elevate immune activity utilizing detoxifying medicine. As the fourth method, high dose of vitamin $C$ therapy will inhibit angiogenesis of cancer tissue and promote increasing of hydro-peroxide in cancer cell [11-12].

\section{Materials and methods}

Post-operative cancer patients are as follows. Gastric cancer: 20, colon cancer: 28 , liver cancer: 3 , breast cancer: 27 , lung cancer: 17, prostate: 11, sarcoma: 2, kidney cancer: 2 , ovarium cancer: 3 , esophageal cancer: 3 , uterine cancer 14, skin cancer: 1, Among them,
104 candidates were contributed to as an intervened group. Others: 27 patients are served to be as a control group. Detoxifying and immunetherapy is composed of intestinal sweeping by detoxifying medicine and Nishi type exercise utilizing Nishi Type apparatus (Orthopedic Traction Apparatus: WA: 57B (686) Yamato (KK, Ehime prefecture, Japan).

As for cancer recurrence prevention, 4 types of intervened method are prepared and applied to 104 candidates: DER, high dose of vitamin C (20g), Herbal medicine (Sun Advance, Chiba city, Japan) and detoxification therapy are applied step by step. As to herbal medicine, SA $1.6 \mathrm{~g}$ was prescribed daily. As for control group (herbal medicine $5 \mathrm{~g}$ as Kudzu Decoction) prescription was applied as placebo. TMCA examination was carried out in everyone month and checked their risk assessment.

Detoxifying therapy is composed of Nishi type [Orthopedic Traction Apparatus : WA: 57B(686) Yamato(KK, Ehime prefecture, Japan)] of exercise for 20 minutes and detoxifying therapy medicine for 6 hours which is sweeping of intestinal canal which eliminate detoxifying medicine [intake of the mixture of citric acid (12g), lactulose ( Kowa, Tokyo) (24g), magnesium hydroxide $(30 \mathrm{~g})$ and $50 \mathrm{ml}$ of water], total body massage, hot and cold water dipping each time for 1 minute alternatively, 7 time repeating) stimulation for autonomic nervous system and sauna bathing at $39^{\circ} \mathrm{C}$ for 20 minutes.

${ }^{*}$ Correspondence to: Tsuneo Kobayashi, MD, PhD, International cancer prevention center, Chiba city, Mihama-ku Takasu 3-21-1, Japan, Tel: +043-3062611; Fax: +043-279-4211; E-mail: ft1992@vega.ocn.ne.jp

Key words: dietary energy restriction (DER), herbal medicine (Sun Advance), high dose of vitamin $C$, detoxifying therapy, recurrence prevention program

Received: April 15, 2018; Accepted: April 26, 2018; Published: May 03, 2018 
At first, I must survey the effectiveness of detoxifying therapy. The following data show that each 3 time of detoxifying therapy decrease the value of CEA, ferritin and alpha ( $\alpha$ )-globulin as alpha( $\alpha)$-immunoregulatory protein in the serum and increase immune activity as the results. T cell number is increasing from 1252 to 2184 .

\section{Results}

At first, I must survey the effectiveness of detoxifying therapy. The following data show that each 3 time of detoxifying therapy decrease the value of CEA, ferritin and alpha $(\alpha)$-globulin in the serum and increase immune activity. T cell number is increasing from 1252 to 2184 .

The effectiveness of detoxifying therapy was showed on 57 years female. (Table 1) that the correlation between immune-suppressive substances and immune activity.

Secondly, intervened treatment group were examined by TMCA in every 1 month. When effectiveness is weak, another intervened method is added step by step. (Figure 1)

The results for 3 years are as follows.

As to control group, aggravation: $55.5 \%$, no change: $33.3 \%$ $3.5 \%$

As to the intervened group; aggravation group: $7.7 \%$, no change:

Thirdly, among them, I have randomly selected one postoperative colon cancer patient (72 yrs.) and followed for 12 years. In this patient, I have intervened by 2 methods: herbal medicine (Sun Advance) and detoxifying treatment therapy. When risk assessment by TMCA become higher risk, detoxifying therapy (in another name is Refreshment therapy) or mix-intervention of herbal medicine (Sun Advance) and detoxifying therapy (Figure 2) were applied. This patient could be prevented his cancer recurrence by these intervened methods for 12 years.

Among the postoperative cancer patients (131), we have carried out intervention treatment by 4 methods which promote natural healing power against higher risk group. We have succeeded in cancer recurrence prevention program in $93 \%$.

\section{Discussion}

As cancer tissue will develop just look like fetus development, we should carry out suitable cancer recurrence prevention program by means which solve cancer nutritional control, solve lowering immune activity, inhibit cancer angiogenesis and solve mitochondria respiratory dysfunction because neoplasm is appeared by immunological decreasing disease. Before clinical neoplasm will be appeared, complementary and alternatively medicine or holistic medicine would be effective for cancer recurrence prevention. In this paper I have failed cancer recurrence prevention in $7.7 \%$. But as to this $7.7 \%$, I have applied immune-thermo-chemotherapy (ITC) [13] on those who have not succeeded in cancer recurrence prevention program after consent. Utilizing ITC, I have succeeded in cancer recurrence prevention in 99 $\%$ ( not published).

In ordinal standard western medicine, $60 \sim 70 \%$ of cancer patients would be recurred up to now. So, my reported recurrence prevention programs are good intervention which are depended upon the activation of natural healing power different from chemotherapy and radiotherapy. We should change the treatment not cancer attacking

Table 1. The effectiveness of detoxifying therapy was confirmed with utilizing tumor marker combination assay, immune-suppressive substances (CEA, ferritin, $\alpha 1$-globulin, $\alpha 2$-globulin) and immune activity ( $\mathrm{T}$ cell number, stimulation index by PHA).

\begin{tabular}{|c|c|c|c|}
\hline Detoxifying therapy & 3 times & 3 times & 3 times \\
\hline Date: & $1983,5 / 9$ & $1983,6 / 2$ & $1983,11 / 4$ \\
\hline CEA (ng/ml) & 3.1 & 2.1 & 1.4 \\
\hline Ferritin (ng/ml) & 203 & 85 & 60 \\
\hline a1-globulin (\%) & 5.7 & 3 & 2.8 \\
\hline a2-globulin (\%) & 11.9 & 8.3 & 8.1 \\
\hline $\mathrm{T}(\%)$ & 73 & 78 & 78 \\
\hline$T$ cell number & 1252 & 1460 & 2184 \\
\hline Stimulation index & 165 & 202 & 152 \\
\hline
\end{tabular}

\begin{tabular}{llllllllllll}
0 & 10 & 20 & 30 & 40 & 50 & 60 & 70 & 80 & 90 & $100 \%$ \\
\hline
\end{tabular}

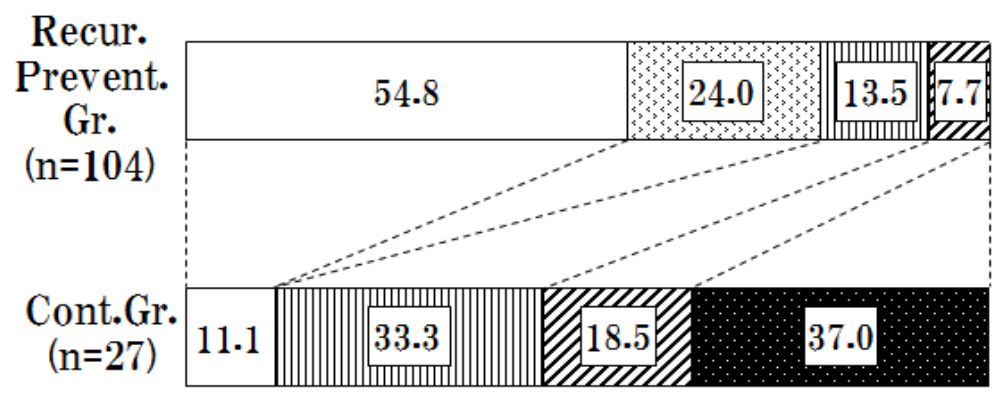

$\square$ Improvement 四 Improving tendency

ש Deteriorating tendency

Deterioration

Figure 1. High risk group (TSIV, and TSV) ( $\mathrm{n}=104)$ are intervened by Herbal medicine (Sun Advance; 1.6g per day) and detoxifying treatment in comparison with control (n=27) Evaluation was carried out by TMCA one time in each month. 


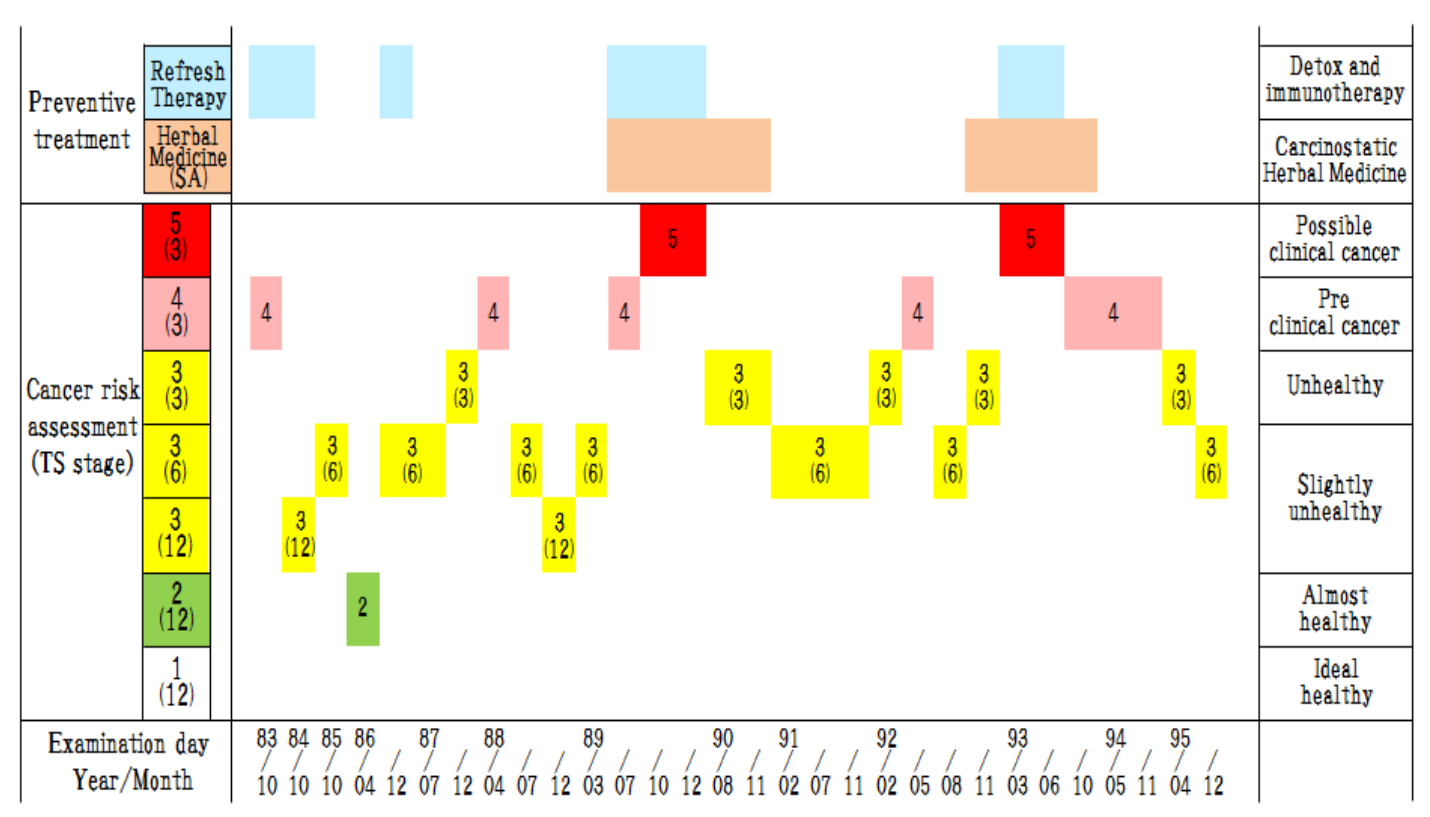

Figure 2. Post -operative colon cancer patient (72 years old) was followed up by the combination intervention of herbal medicine and detoxifying treatment.

invasive treatment but contributed to promote natural healing power. Most of cancer sacrifices are derived from cancer recurrence in $60 \sim 70 \%$. If our cancer recurrence prevention program is applied to the postoperative patients with high risk, cancer patients will be secured.

\section{Funding}

There is no funding in this paper.

\section{Acknowledgements}

I am grateful to my co-worker: Hayashida Shgeru.

\section{Conflict of interest}

No confliction.

\section{References}

1. Kobayashi T, Kawakubo T (1994) Prospective investigation of tumor markers in early cancer screening and risk assessment. Cancer 73: 1946-1953. [Crossref]

2. Seyfried TN (2011) Mitochondrial glutamine fermentation enhances ATP synthesis murine fibroblastoma cell. Processing of $102^{\text {nd }}$ Annual Meeting of the American Cancer Research, 2011, Orland FL.

3. Warburg O (1969) Revised Lindau Lectures. The prime cause of cancer and prevention. Parts 1\&2, In: Burk D. editor Meeting of the Nobel Laureates Lindau, Lake Constan Germany: K. Triltsch.

4. Reitzer LJ, Wice BM, Kennel D (1979) Evidence that glutamine, not sugar is the major source for cultured Hela cells. J Biol Chem 254: 2669-2676. [Crossref]
5. Onogi N1, Okuno M, Komaki C, Moriwaki H, Kawamori T (1996) Suppressing effect of perilla oil on azoxymethane-induced foci of colonic aberrants in rats. Carcinogenesis 17: 1291-1296. [Crossref] $\square$

6. Campbell TC, Cheng J (1994) Diet and chronic degenerative diseases. A summary of results from an ecologic study in rural China. In NJ Teple and DP Burkitt(eds). Western diseases: their dietary prevention and reversivility 67-118. Totowa N.J Humana Press.

7. De Stavok BL, Wang DY, Allen DS (1993) The association of height, weight, menstrual and reproductive vents with breast cancer. Results from two prospective studies on the island of Guernsey (United Kingdom). Cancer causes and control 4: 331-340.

8. Youngman LD (1990) The growth and development of aflatoxin B1-induced preneoplastic lesions tumors, metastasis and spontaneous tumors as they are influenced by dietary protein level, type and intervention. Ithaca NY: Cornell University $\mathrm{PhD}$ Thesis.

9. Tanimizu T, Sugimoto K, Hayashi N, Miura M, Kijima C, et al. (1982) New approach to Chinese herb medicine, inhibition by Chinese herb medicine" Sun Advance" of SV40transformation in mouse cells. Proc. Symposium. WAKAN-YAKU 15: 228-33.

10. Sugimoto K, Jo T, Tanimizu T, Hayashi N, Dogome M, et al. (1982) The effects of antitumor herb medicine "Sun Advance" in mice. Proceeding Symposium. WAKANYAKU 15: 224-227.

11. Chen Q, Espey MG, Krishima MC, Mitchell JB, Corpe CP, et al. (2005) Pharmacologic ascorbic acid concentration selectively kill cancer cells, Action as prodrug to delivery hydrogen-peroxide to tissue. Proc Natl Acad Sci U S A 102: 13604-13609. [Crossref]

12. Chen Q, Espey MG, Sun AY, Lee JH, Krishna MC (2007) Ascorbate in Pharmacologic concentration selectively generates ascorbate radical and hydrogen peroxide in extracellular fluid in vitro. Proc Natl Acad Sci U S A 104: 8749-8754. [Crossref]

13. Hayashida S, Sugimoto K, Kobayashi T (1984) Effect of BRM-induced systemic hyperthermia combined with immunochemotherapy on advanced breast cancer metastasis. $5^{\text {th }}$ International Symposium on hyperthermic Oncology: 361.

Copyright: (2018 Kobayashi T. This is an open-access article distributed under the terms of the Creative Commons Attribution License, which permits unrestricted use, distribution, and reproduction in any medium, provided the original author and source are credited. 